\title{
Long-term and intra-day variability of BL Lacertae since the last great outburst
}

\author{
Maria G. Nikolashvili and Omar M. Kurtanidze
}

Abastumani Observatory, 383792 Abastumani, Georgia

\begin{abstract}
We present the results of optical photometry of BL Lacertae carried out using ST-6 CCD camera attached to the Newtonian focus of the $70 \mathrm{~cm}$ meniscus telescope of Abastumani Observatory. On the basis of observations conducted since August 1997 during more than 550 nights about 17000 frames were collected. They have been reduced using Daophot II.

It has been shown that optical variability of BL Lacertae is very complex. The maximum variation was observed at long-term scale and is equal to equals to $3.0 \mathrm{mag}(\mathrm{rms}=0.03)$ in $\mathrm{B}$ band, while the variation in $\mathrm{V}$ and $\mathrm{R}$ bands are within $2.71 \mathrm{mag}(0.02)$ and $2.53 \mathrm{mag}(0.01)$, respectively. This means that variations are larger at shorter wavelength or the object become bluer in the active phase. It were also demonstrated that BL Lacertae shows intra-day variability within $0.30 \mathrm{mag}$ (0.02), while intra-hour variability within $0.10 \mathrm{mag}$ (0.01) magnitudes.
\end{abstract}

Keywords. BL Lacertae objects - CCD photometry - variability

\section{Introduction}

BL Lacertae is a prototype of one of the most extreme subclass of AGNs. It was discovered in 1929 by Cuno Hoffmeister, who found it to vary by more than a factor two in one week and classified it as a short period variable star (Hoffmeister 1990). Since its identification as an extragalactic source it was the subject of numerous studies in many frequency bands. Historically, BL Lac is known to show 5.0 mag variation in optical band with episodic outbursts (Maessano 1997). Maximum variation in the infrared $\mathrm{K}$ band is 3.0 mag (Fan 1999).

During the summer 1997 outburst BL Lac exhibited a very strong activity (Clements 2001). The strong activity was also detected in the radio, X-ray and gamma-ray bands (Sambruna 1999; Madejski 1999; Tanihata 2000; Bottcher 2000).

\section{Observation and data reduction}

We have been intensively monitoring BL Lacertae at Abastumani Observatory since 1997, when it remained in a high state for more than two months. Rapid and large amplitude flux variations characterized the source during this period. Here we present observations carried out from August 1997 to November 2001. All observations were carried out with 70-cm meniscus telescope and CCD camera ST-6 attached to the Newtonian focus $(1 / 3)$. To study the long-term variability we observed BL Lacertae during 317 nights, collected 320 frames in each of the BVI bands, and 465 frames in the R band. More than 16000 frames were obtained in $\mathrm{R}$ band during 259 nights to study intra-day variability (Wagner 2001) as well as intra-hour variability (Miller 1989). The duration of observational runs varied from two hours to six hours. The exposure times varied from 60 to $180 \mathrm{sec}$ depending on the brightness of the object and the filter used. Instrumental differential magnitudes were calculated relative comparison stars $\mathrm{C}$ and $\mathrm{H}$, that have nearly the same colours as the object under study (Smith 1985). The images were reduced using 
Daophot-II (Stetson 1987). The highest differential photometric accuracy reached in $\mathrm{R}$ band is $0.005(\mathrm{rms})$ magnitude at $m(r)=14.00 \mathrm{mag}$ during $180 \mathrm{sec}$. Magnitudes were calculated relative comparison stars $\mathrm{C}$ and $\mathrm{H}$, that have nearly the same colours as the object under study. To eliminate the effects of seeing induced spurious IDV and IHV (Cellone 2000) the apertures are taken to include the whole host galaxy.

\section{Results and conclusions}

The constructed long-term variability lightcurves have shown that maximum B-band variation was recorded during August 1997 and it amounts to $3.0 \mathrm{mag}$ ( $\mathrm{rms}=0.03$ ), while the maximum amplitude in $\mathrm{R}$ band equals $2.53 \mathrm{mag}$ (0.01). The amplitudes of variation in $\mathrm{R}$ band of the other observing seasons are presented in the table. The results of optical observations of BL Lacertae during great summer 1997 outburst have been presented by different blazar monitoring groups (Speziali 2000; Fan 2001; Clements 2001; Villata 2002, Villata 2004). On the basis of observations of BL Lacertae during the period from August 1997 to November 2001 it was clearly demonstrated that variations are larger in B band or the object become bluer in the active phase (Nikolashvili 1999; Kurtanidze 2001; Nesci 2001), that were also confirmed by other groups (Clements 2001; Fan 2001).

The significant statistical evidences of intra-day and intra-hour variabilities are found during many nights of observations. The typical intra-day and intra-hour variability amplitudes in R band are within 0.30 (0.02) and 0.10 (0.01) magnitudes, respectively. Detailed study of BL Lacertae during multi-wavelength campains carried out in the frame of WEBT collaboration are most extensive study of this prototype source ever conducted (Villata 2004).

\section{Acknowledgements}

O.M.K. gratefully acknowledges invaluable financial support and the kind collaboration with Dr. G. M. Richter without which this Programme would have never been conducted.

\section{References}

Bottcher, M. \& Bloom, S. D. 2000, AJ, 119, 469

Celone, S. A. et al. 2000, AJ, 121, 90

Clements, S. D. \& Carini, M. T. 2001, AJ, 119, 534

Fan, J. H. et al. 1999, AAS, 133, 217

Fan, J. H. et al. 2001, A\&A, 369, 758

Hoffmeister, G. et al. 1990, Veranderlichte Sterne, Leipzig

Kurtanidze, O. M. et al. 2001, in: S. Ritz et al. (eds.), 6th Compton Symp. (AIP CP), 587, 333

Madejski, G. M. et al. 1999, ApJ, 521, 145

Maessano, M., et al. 1997, AAS, 122, 267

Miller, H. R. et al. 1995, Nature, 337, 627

Nikolashvili, M. G. et al. 1999, in: Blazar Monitoring Towards the 3rd millenium, C. M. Raiteri, et al. (eds.), (Osserv. Astron. di Torino: Torino), p. 33

Sambruna, R. M. et al. 1999, ApJ, 515, 140

Smith, P. S. et al. 1985, AJ, 90, 1184

Speziali, R. \& Natali, G. 2000, AAS, 339, 382

Stetson, P. B. 1987, PASP, 99, 191

Tanihata, S. et al. 2000, ApJ, 543, 124

Villata, M. et al. 2002, A\&A, 390, 407

Villata, M. et al. 2004, A\&A, 424, 497

Wagner, S. J. 2001, ASP CS, 227, 112 